\title{
FILOSOFÍA DE LA HISTORIA Y ÉTICA DEL FUTURO
}

\author{
Johannes Rohbeck \\ Technische Universität Dresden
}

\begin{abstract}
The themes "philosophy of history" and "ethics of the future", suggest that the responsibility to future generations should be understood as a concrete problem of philosophy of history. The argument implies a hypothesis that suggests that the future, as such, is history, or which belongs to the history. The concept is obviously nonsense, since we understand history as an entity that includes the events have already occurred. This is the case of historiography, which gathers and presents on a discursive level, past events. However, to deal with the future and ethical reflection as a specific problem of the philosophy of history, despite the foregoing, I shall here through an understanding of the distinction between strong and weak understanding of the philosophy of history.
\end{abstract}

Keywords: Ethics of the future, philosophy of history, responsibility, Jonas, Birnbacher.

Resumo: Os temas "filosofia da história" e "ética do futuro", inferem que a responsabilidade para com as futuras gerações deve ser entendida como um problema concreto da filosofia da história. $\mathrm{O}$ argumento implica uma hipótese que sugere que o futuro, como tal, é história, ou que pertence à própria história. $\mathrm{O}$ conceito é, obviamente, um contra-senso, uma vez que entendemos a história como uma entidade que inclui os eventos já ocorreram. Este é o caso específico da historiografia, que reúne e apresenta em um nível discursivo, eventos passados. No entanto, para tratar do futuro e da reflexão ética como um problema específico da filosofia da história, apesar do exposto, procederei aqui por meio da distinção entre um entendimento forte e um fraco entendimento da filosofia da história.

Palavras-chave: Ética do futuro, filosofia da história, responsabilidade, Jonas, Birnbacher.

Los temas »filosofía de la historia« y »ética del futuro«, infieren que la responsabilidad hacia las generaciones futuras ha de ser comprendida como un problema concreto de la filosofía de la historia. El argumento implica 
una hipótesis que sugiere que el futuro como tal es historia, o bien, que pertenece a la historia misma. El concepto es obviamente contra intuitivo, dado que comprendemos a la historia como un ente que engloba los acontecimientos ya acontecidos. Éste es el caso específico de la historiografía, la cual reúne y presenta, a un nivel discursivo, los hechos pasados. Sin embargo, para tratar al futuro y a la reflexión ética como un problema concreto de la filosofía de la historia, a pesar de lo mencionado, distingo entre una comprensión fuerte y una comprensión débil de la filosofía de la historia.

Podemos incluir al futuro, en un sentido débil, dentro del ámbito de la filosofía de la historia, aplicando su método, limitado hasta el día de hoy en el análisis de eventos pasados, sobre los discursos del futuro. Con esta operación reconocemos que una vista abierta hacia el futuro también logra describir acontecimientos y procesos sociales, cuyas estructuras se pueden analizar. Es claro que el tiempo también juega un rol en el futuro, de manera que el arsenal analítico de estructuras de tiempo y la semántica de tiempos históricos devienen a ser aplicables dentro de esta área. Además, en tanto que podemos afirmar que los acontecimientos futuros son acontecimientos narrados, que los discursos de futuro también son narrativas, o sea que presentan una estructura narrativa, se pueden aplicar las teorías narrativas sobre la representación narrativa del futuro. La filosofía de la historia puede jugar un rol muy particular en un tal análisis narratológico, siempre y cuando sea posible comprobar que dentro de las narrativas específicas del futuro surtan efecto ciertos patrones de interpretación, propios de la filosofía de la historia. Entre estos patrones de interpretación podemos mencionar los modelos de progreso, modelos cíclicos, teleológicos y así seguido.

En un sentido fuerte podemos afirmar que el futuro mismo es historia. El futuro es historia a un nivel substancial, de la misma manera que el pasado y el presente lo son. Desde un punto de vista ontológico, podemos decir que el tiempo no solo es tiempo futuro, sino también historia futura. Una objeción que intenta afirmar que solamente los acontecimientos pasadas se pueden narrar, se ve debilitada ante lo dicho, ya que los discursos del futuro también contienen una estructura narrativa. Sin embargo, mi argumento central sostiene que la historicidad del futuro se ha de comprender partiendo de un punto de vista de práctico. No es suficiente comprender a la historia como una narración, las acciones se orientan hacia el futuro, creando de esta manera tiempo e historia. 
Yo vinculo el teorema presentado explícitamente a la filosofía de la ilustración de la historia. No es sino hasta los comienzos del siglo XVIII que se concibió una continuidad entre pasado, presente y futuro que posibilitaba (de manera similar al concepto de historia) un concepto generalizado del »futuro«. Visto desde una perspectiva del progreso, esta filosofía de la historia se orientaba no solo en la acción, sino también, de manera programática, en el futuro. Es así que a la escatología cristiana le prosiguen dos alternativas: la teleología de la historia (Turgot y Kant) y la prognosis racional (Condorcet). Mientras que el historismo del siglo XIX se mantenía fijado en el pasado, el siglo XX se ocupó con las catástrofes del presente. Solo en ciertos casos logro resurgir el pensamiento utópico de la ilustración y del marxismo.

Sin embargo, puedo hacer referencia en este punto a Heidegger, quién comprendió el futuro de la persona decidida a tomar una acción, sobre todo en la "pena" (Sorge), como la historia "propia« (eigentliche). La labor del historiador consta entonces en interpretar tales proyectos futuros, realizados en el pasado, como hechos consumados. Paul Ricœur exigió en su obra tardía, en continuación a Heidegger y de manera casi contradictoria a su teoría narrativa de Tiempo y narración, posicionarse en la perspectiva del ser humano antecedente, para poder realizar con ello, de una manera justa, sus planes e intenciones. El tema central de un futuro pasado, como lo elabora Reinhart Koselleckt, también infiere un entendimiento que comprende a las acciones pasadas como proyectos de futuro. Además, los conceptos centrales de un espacio de experiencia y un horizonte de expectativa, implican, por un lado, que las experiencias hechas en el pasado influyen a la expectativa del futuro, y por el otro lado, que la expectativa del futuro le va dando forma a nuestra interpretación de la historia acontecida. Todas estas posiciones implican un concepto más general de historia, que engloba en si al pasado, al presente y al futuro.

Con esta argumentación quiero hacer claro que me siento inclinado tanto hacia la comprensión débil, como la interpretación fuerte de un futuro comprendido como historia. La variante débil es en realidad simplemente una teoría de historia, ya que no realiza más que un traslado de su teoría científica, ó de su metodología, sobre los discursos de futuro que analiza. La variante fuerte ha de ser comprendida como una filosofía de la historia, puesto que se coloca sobre una base de acción que encuentra su directriz en el futuro, posicionándose con ello al mismo tiempo dentro del ámbito de la filosofía práctica. Todo ello esclarece la relación que hay entre filosofía de la 
historia y ética del futuro. Es de esta manera que se ha de comprender correctamente el título de mi presentación.

Para elaborar la dimensión ética de una filosofía de la historia, orientada hacia el futuro y fundada en la teoría de acción, es necesario crear un puente hacia la ética del futuro. Dicha ética ya presenta un establecido discurso de la ética aplicada. En la ética del futuro se gestionan preguntas con respecto a la responsabilidad hacia las generaciones venideras. Es claro que con ello es importante considerar las relaciones temporales - no solo la relación entre presente, pasado y futuro como tales, sino también los tiempos entre generaciones cercanas y lejanas, ante las cuales hemos de tomar la responsabilidad con respecto a nuestras acciones.

Sin embargo, en los debates actuales sobre la responsabilidad duradera se ven frecuentemente desapercibidos los temas del tiempo y la historia. Dado a que considero la reflexión filosófica sobre dicho problema un desiderátum importante en la filosofía de la historia, intento demostrar que el análisis de la dimensión específicamente histórica de la responsabilidad, es imprescindible para poder completar la argumentación ética. Ello significa para la ética del futuro una apertura hacia preguntas normalmente propuestas en la filosofía de la historia, obteniendo con ello la oportunidad de integrar ciertas conocimientos de la estructura y la función de la conciencia histórica en su fundamento. Para la filosofía de la historia significa una forma de participar en la solución de ciertos problemas de la filosofía practica que requieren de una perspectiva del futuro. La meta esta entonces en realizar un enlace entre la filosofía de la historia y la ética del futuro.

Para empezar analizaré de manera crítica las estructuras de tiempo inherentes a una ética del futuro. Con ello intentare demostrar que ciertas éticas se deciden por prescindir del problema del tiempo por razones meramente sistemáticas. Hay filósofos que lamentan un olvido del futuro en la ética. Yo le confirmo a la ética del futuro contemporánea un olvido general del tiempo. Desde esta perspectiva práctica y en contra del problema mencionado, intento fundar la responsabilidad duradera en el contexto de una filosofía de la historia. Con el propósito de vincular la ética con la filosofía de la historia proseguiré en dos pasos. En primer lugar, presentaré un fundamento general que se enfoca en la inevitabilidad del presente y la omnipresencia del cambio histórico; finalmente nombraré modelos concretos 
que considero adecuados para construir un relación efectiva entre los tiempos lejanos y cercanos que atañen a la responsabilidad.

\section{Filosofía ética de la historia y la historización de la ética}

Con el concepto de una "filosofía de la historia" no me refiero al paradigma en su totalidad, sino, en este caso, a la reflexión filosófica sobre ciertos aspectos históricos de la ética. Eso no significa que no podemos llegar a actualizar ciertos elementos de la >clásica< filosofía de la historia, así como motivos del historismo y otras tendencias de esta rama filosófica. En una retrospectiva de tal índole podemos ver que la filosofía de la historia y la ética no siempre han surgido como materias separadas, como lo es el caso de la ética contemporánea. Desde la época de la ilustración europea se fueron entrelazando ambas disciplinas. Por un lado, porque la filosofía de la historia presentaba ciertos planteamientos para una ética del futuro, y, por el otro lado, porque la ética se vio temporalizada con el surgimiento de la conciencia histórica. En este sentido podemos hablar de una filosofía ética de la historia y una historización de la ética. Con respecto a este relación quiero introducir la propuesta de una síntesis entre la filosofía de la historia y la ética.

El preludio hacia una explicita ética del futuro lo presentó en Alemania Hans Jonas con su obra El principio de responsabilidad. Esta obra hace referencia, ante todo, a las generaciones futuras. ${ }^{1}$ Jonas temporalizó radicalmente la ética, aplazando el espacio temporal de la responsabilidad hacia el futuro. La razón para ello la veía en una nueva dimensión en la »teledinamia« de las tecnologías modernas, que exigían de la responsabilidad un mayor "alcance en el futuro«. Teóricos contemporáneos como Dieter Birnbacher, en su obra Responsabilidad para generaciones futuras, se basan en el concepto de una responsabilidad duradera como la presentó Jonas. Dado que el poder de disposición técnica se extiende hasta el futuro y llega a poner en juego la existencia del ser humano, es necesario introducir esta nueva disciplina de una ética del futuro, capaz de lograr una extensión del horizonte del tiempo. ${ }^{2}$ En conformidad con el utilitarismo se habría de hallar la solución en un balance completo de los intereses actuales y futuros.

1 JONAS, H. Das Prinzip Verantwortung, Frankfurt a. M. 1979, P. 9, 39, 64, 84, 220.

2 BIRNBACHER, D. Verantwortung für zukünftige Generationen, Stuttgart 1988, 11 ff. 
No obstante, en tiempos recientes las oportunidades para una realización práctica de la ética del futuro se contemplan con mayor escepticismo. El rebasamiento del horizonte espacio-temporal nos confronta con el peligro de que el nuevo tipo de responsabilidad se mueva en una zona incierta y sin compromiso. ${ }^{3}$ Por una parte, si presuponemos una relación de experiencia entre las generaciones, la responsabilidad ante los seres humanos de tiempos por venir aparece como una osadía. ${ }^{4} \mathrm{Si}$ presuponemos, por otra parte, personas endilgadas por intereses en vez de generaciones, se presenta el problema de una sobreexigencia. ${ }^{5}$

El problema esencial de la responsabilidad duradera consiste, a mi juicio, en la omisión del presente y en el traslado de la responsabilidad hacia generaciones futuras directo hacia el futuro. El argumento presentado sostiene que un sujeto debe ser responsable a distancia para poder responder en el futuro de manera retrospectiva ante sus rendimientos y errores cometidos en el presente. ${ }^{6}$ Se deduce entonces la responsabilidad prospectiva a partir de la mirada retrospectiva que presupone una anticipación del futuro. En lugar del criticado presentismo, acusado de preferir los intereses contemporáneos a expensas de los futuros, se opone aquí un futurismo ingenuo que exige tomar en serio el destino de las personas futuras observandolas como convivientes contemporáneos.

Detrás de este dilema encontramos un universalismo ético que ultima en una abstracción del tiempo. Ello resulta de una »evaluación del futuro« que le atribuye el mismo valor tanto a los intereses y bienes futuros como a los presentes. De la constatación de que todos los seres humanos tienen los mismos derechos, independientemente del tiempo en que viven, se deduce una equivalencia de todos los tiempos. Cualquier tipo de preferencia

3 GETHMANN, C. F. Langzeitverantwortung als ethisches Problem im Umweltstaat. In: Langzeitverantwortung im Umweltstaat, Bonn 1993, 7; HUBIG, C. Langzeitverantwortung im Lichte provisorischer Moral. In: MITTELSTRASS, J. (Hg.). Die Zukunft des Wissens, Berlin 2000, 298 ff., insbes. 302.

4 LEIST, A. Ökologische Gerechtigkeit. Global, intergenerationell und humanökologisch. In: NIDARÜMELIN, J. (Hg.) Angewandte Ethik. Die Bereichsethiken und ihre theoretische Fundierung. Ein Handbuch, 2. Aufl. Stuttgart 2005, 425-513; $19 \mathrm{f}$.

${ }_{5}^{5}$ STURMA, D. Die Gegenwart der Langzeitverantwortung. In: LANGBEHN, C.(Hg.). Recht, Gerechtigkeit und Freiheit, Paderborn 2006, S. $221 \mathrm{ff}$.

${ }^{6}$ HUBIG, a.a.O., 26. 
temporal cae así bajo sospecha de querer defender intereses presentes. ${ }^{7}$ Del mismo modo en que el ético debe mantener una posición imparcial, se exige que la relación entre los tiempos mantenga una "imparcialidad cronológica«. La posición ética se distingue, de este modo, en el hecho de que prescinde del tiempo; es por ello que una ética universalista implica también una abstracción del tiempo. Este tipo de ética del futuro es una ética sin tiempo, y por ello consecuentemente también sin tiempo futuro.

Para compensar esta abstracción del tiempo sirven construcciones ficticias que pretenden hacer calculable la relación entre seres contemporáneos y futuros. La variante utilitarista consiste en imputar ciertos intereses y derechos a las generaciones "lejanas«, percibidas de manera representativa por personas existentes hoy en día. Todo ello para establecer una suma intergeneracional de beneficios. ${ }^{8}$ La variante teórica del discurso, por su parte, construye un dialogo ficticio entre las generaciones presentes y futuras. El vínculo con la humanidad futura lo aporta la "anticipación de una comunidad discursiva sin plazo fijo «. ${ }^{9} \mathrm{El}$ concepto más difundido en tal perspectiva es el de un »contrato generacional«, tal y como lo presenta la teoría de la justicia de John Rawls. La metáfora del »velo de la ignorancia« se modifica de tal manera que logra incluir la ascendencia temporal de los implicados en el área de la ignorancia. También en este caso nos encontramos con la "congregación simultanea de toda generación pensable ${ }^{10}$ La fortaleza de este tipo de construcciones radica, sin duda alguna, en su reivindicación universal de validez, la cual, posibilita extender el principio de responsabilidad a generaciones lejanas. Pero los límites que posee, ya nombrados, se presentan en una motivación carencial que hace de la obligación lejana una apelación débil.

El comunitarismo presenta una alternativa en este campo de juego. Sus representantes critican el utilitarismo abstracto, así como el contratismo hipotético. El comunitarismo se enfoca en las relaciones concretas entre las (tres) generaciones convivientes que ya contienen en sí elementos para una responsabilidad futura. Los padres, por ejemplo, muestran un interés

7 BIRNBACHER 1988, a.a.O., 29, 35; mit Berufung auf Hare ders. 2001, a.a.O., 124; kritisch zu Birnbacher siehe Gethmann/Kamp, a.a.O., 147; vgl. Leist, a.a.O., 26.

${ }^{8}$ BIRNBACHER 1988, a.a.O., $101 \mathrm{ff}$.

9 GETHMANN, a.a.O., 12.

10 CASPAR, a.a.O., 96; LEIST, a.a.O., 19. 
elemental hacia sus hijos, de los que se deben encargar. El principio clave en una tal ética es el del cuidado familiar. ${ }^{11}$ Ciertamente, hay un logro en esta responsabilidad hacia el semejante cercano, consistente en el nexo de vida prácticamente experimentado, percibido y motivado. Ahora bien, al mismo tiempo se torna perceptible el límite temporal entre las generaciones, imposible de rebasar sobre la angosta base moral del cuidado paternal. Aquí emerge la pregunta acerca de cómo transferir la dimensión práctica de las personas por venir al horizonte de un futuro lejano.

En el contexto constructivista se propone tomar la responsabilidad hacia personas en el futuro cercano, por lo menos como punto de partida para la fundación de una responsabilidad duradera. Y ello con el propósito de extender la obligación cercana hacia el futuro lejano. ${ }^{12}$ Sin embargo, no podemos justificar éticamente un »descuento" de los intereses y bienes futuros, de ello no podemos deducir que existe una falta de diferencia entre obligación cercana y obligación lejana. No es indiferente si padres cuidan de sus hijos o si se preocupan por otras innumerables generaciones. Para reproducir esta imagen de vida sin caer en la trampa de un descuento, se introduce el termino de gradualidad. Evitando así el universalismo y reteniendo simultáneamente la responsabilidad duradera. Bajo esa premisa vale construir la obligación lejana partiendo de la cercana, deduciendo de la experiencia con el futuro cercano una conducta moral respecto al futuro lejano. La meta es presentar una relación concreta entre las generaciones cercanas y lejanas según el modelo del cuidado. El programa metódico se llama: extrapolación constructiva.

\section{El presente de la obligación lejana}

Luego de haber analizado críticamente las precondiciones implícitas en las estructuras temporales que se manejan en las éticas del futuro, continuaré en la línea del fundamento sistemático. Considero esencial la reflexión sobre el tiempo y la historia, porque la dimensión histórica representa la relación del ser humano contemporáneo con una praxis social. Desde esta perspectiva práctica intento fundar la responsabilidad duradera en

${ }^{11}$ LEIST, a.a.O., 20, 28; CASPAR, a.a.O., 92 f.; JONAS, a.a.O., 197 f.

12 GETHMANN, a.a.O., 11, 15; GETHMANN/KAMO, a.a.O., 145, 149; vgl. STURMA, a.a.O., 235. 
el contexto de una filosofía de la historia. Con el proposito de vincular la ética con la filosofía de la historia proseguiré en dos pasos. En primer lugar, presentaré un fundamento general que se enfoca en la inevitabilidad del presente y la omnipresencia del cambio histórico; finalmente nombraré modelos concretos que considero adecuados para construir un puente realista entre los tiempos lejanos y cercanos que atañen a la responsabilidad.

Un beneficio epistémico que obtenemos con ello en el contexto de la filosofía de la historia, consiste en que la responsabilidad solamente se puede relacionar con personas vivientes. Sólo partiendo de esta perspectiva presente es posible deducir una responsabilidad lanzada hacia el futuro. La inevitabilidad del presente constituye el trascendental de cualquier ética del futuro.

Denomino trascendental esta relación con el presente para aclarar que se trata de un principio capaz de reivindicar validez más allá de una discusión de intereses. ${ }^{13}$ La identificación de una posición en el presente con una preferencia por los intereses actuales no es forzosa, de la misma manera que la abstracción del presente o del tiempo no es garante de decisiones morales correctas. Es por ello que la reflexión sobre la dimensión temporal ha de ser igual de imparcial que el universalismo ético. Reconociendo la perspectiva del presente se nos abre el campo de debates sobre la distribución justa de bienes futuros.

Ahora bien, la presencia de la responsabilidad lejana se ve acompañada de consecuencias. Tomando las personas actuales, con sus discusiones controvertidas, como punto de partida, se puede evitar fácilmente las sobreexigencia moral. Una presupuesta constelación de intereses ofrece al mismo tiempo una mejor oportunidad para motivar a las personas hacia la acción práctica. La primacía del presente significa construir una relación lo más realista posible entre la vida presente y las generaciones venideras.

Otra consecuencia consiste en considerar la singularidad del presente como un tiempo histórico especial. De ello se deduce que es imprescindible contar con un concepto del futuro en su calidad de ser diferente. Por ello no es suficiente proyectar el desarrollo actual de la civilización técnico-científica

${ }_{13}$ Para la diferencia entre las concepciones de obligación y responsabilidad vease: HEIDBRING, L. Grenzen der Verpflichtung. Zum Verhältnis von Verantwortung und Pflichten. In: LANGBEHN, C. (Hg.) Recht, Geerechtigkeit und Freiheit, Paderborn 2006, 239-268. 
hacia el futuro para luego admitir a lo sumo dificultades en el pronóstico realizado. Para poder encontrar un juicio ético es decisivo reconocer el hecho de que en el futuro cambiarán, sobre todo las escalas de valores de las personas.

Éticas del futuro utilitaristas realizan en el fondo una continuación de la idea del progreso a la hora de atribuirle a las futuras generaciones tecnología nuevas e imprevisibles, suponiendo un mantenimiento simultaneo de intereses y necesidades constantes. Proponen, de esa manera, la posibilidad de poder realizar en cualquier momento una suma de beneficios partiendo de los bienes y las exigencias que se requieren para la felicidad. En contra de ello podemos decir que la idea de una buena vida buena obtiene un sentido solamente en el contexto de una comprensión genuina de lo temporal, lo cual implica que el tiempo está acompañado siempre de rupturas históricas. ${ }^{14}$ Muchos utilitaristas empiezan a unirse a esta convicción, complementando la inseguridad en el pronóstico de una evaluación de efectos técnicos con una inseguridad con respecto una transformación de las metas futuras. De acuerdo con ello es de esperar entonces, que la cultura se desarrolle en dirección a una "comunidad de valores" que motiva constantemente nuevas posibilidades en tiempos venideros. ${ }^{15} \mathrm{~A}$ la hora de articular con ello el concepto de un "futuro abierto", se hace manifiesta una conciencia histórica mejor diferenciada.

Partir de un principio abierto en el transcurso histórico conduce a no colocar bienes específicos como un elemento primordial en la ética del futuro, dado que el valor futuro de estos es incierto. Lo apropiado sería crear condiciones para que se abra la posibilidad misma de una disposición de bienes necesarios. ${ }^{16}$ Tampoco se trata de una preocupación por intereses establecidos que pudiesen tener personas futuras, sino del desarrollo y conservación de condiciones económicas, sociales y culturales razonables para una vida digna. La meta es la de mantener abierto el horizonte de posibilidades en el que se mantienen y crean áreas de juego para la acción y para la realización de concepciones de vida.

\footnotetext{
${ }^{14}$ Crítico de BIRNABACHER: VOSSENKUHL, a.a.O., 49.

${ }^{15}$ BIRNBACHER/SCHICHA, a.a.O., 19 f., 24; LEIST, a.a.O., 29; STURMA, a.a.O., 230.

${ }^{16}$ BIRNBACHER/SCHICHA, a.a.O., 19 f., 23; STURMA, a.a.O., 230; LEIST, a.a.O., 29; CASPAR, a.a.O., 100.
} 


\section{Modelos de responsabilidad duradera: la generación y la herencia}

En este último paso del intento de una fundación sistemática nombraré algunos modelos que considero apropiados para crear un puente entre el presente y el futuro.

El modelo constructivita ya mencionado contiene una contradicción. Presenta un modelo de cuidado que es necesario superar. Cuando el cuidado de los padres es limitado a tres generaciones, es necesario encontrar otro modelo que permita crear continuidades entre los tiempos cercanos y lejanos de la responsabilidad. Se ha de buscar un modelo que no sea tan cercano al tiempo inmediato como el modelo del cuidado, pero sí mas concreto que la idea de una comunidad de discurso anticipada, que la de una balanza intergeneracional de beneficios o que la de un contrato entre generaciones. Propongo como solución un modelo que procede del área de la historia: el modelo de herencia en la sucesión de generaciones.

Para introducir el modelo de la herencia explicaré previamente el concepto de una sucesión de generaciones. Para evitar quedarse limitados en una visión de herencia biológica es importante entender el concepto de un legado de cada generación como una herencia cultural.

\section{Sucesión de generaciones}

Transfiriendo la pregunta acerca del sujeto de la historia al ámbito la ética del futuro, obtendríamos para su respuesta tres candidatos: el género, la generación y la persona. En los primeros pasos de la ética del futuro se postuló la responsabilidad como orientada fundamentalmente hacia la humanidad futura, y consecuentemente se produjo una fuerte valoración de la continuidad del género humano; de la misma manera que el problema de su exterminio se consideraba como una catástrofe moral. ${ }^{17}$ Las éticas del futuro posteriores se retiraron de este patetismo. Podemos notar la naturalidad con la que se habla sobre generaciones en las éticas posteriores, sin que el término sea reflejado de una manera estrictamente filosófica. En contra de esta posición, apareció una crítica que no reconoce significado

17 JONAS, a.a.O., 86, 245; BIRNBACHER 1998, a.a.O., 102. 
ético y social al término de la generación. Esta critica introduce el concepto de persona como único sujeto y destinatario de la responsabilidad. ${ }^{18}$ Considero al mismo tiempo apropiado utilizar el término de la generación, lo cual propicia una mediación de la obligación cercana y la obligación lejana. Con ese fin intento reformular dicho concepto en el sentido de una categoría importante para la filosofía de la historia y que contendría los siguientes tres aspectos.

Primero: el término de la generación media entre naturaleza y cultura. ${ }^{19}$ El término "generatio« significa, por un lado, la procreación en el sentido de la reproducción natural. Fijando el tiempo de una generación en treinta años, obtenemos para la obligación cercana de un adulto un periodo de aproximadamente cincuenta años. Aquí topamos con una medida biológica sin una determinación filosófica consistente. El concepto de generación tiene, por el otro lado, un significado cultural, como lo documenta la investigación de generaciones en la ciencia histórica. Por poner unos ejemplos, podríamos mencionar, entre los temas actuales de esta ciencia, el de la generación de la guerra o el de la generación del 68, entre otros muchos. ${ }^{20}$ Lo importante en nuestro contexto es ver que todo ello esta directamente relacionado con la expectativa característica de un concepto de cultura. Sin importar lo impreciso que pueda ser, son susceptibles de servir para establecer una mediación entre una preocupación familiar y una responsabilidad duradera.

Segundo: El término generación tiene la ventaja de encontrarse en un punto medio entre las ideas de humanidad y de individuo. Por un lado, evade el macrosujeto de un genero humano, decisivo en la filosofía de la historia desde la ilustración hasta Hegel, y despedido con razón en el seno del historismo. Después de que el sujeto de referencia "genero« se haya mostrado como sobredimensional, el concepto de generación se hace más prometedor, en la medida en que alude a poblaciones perceptibles y variadas. Además, el concepto evade, por otro lado, la fijación del problema ético en el ámbito de personas individuales, las cuales, a pesar de ser actores

${ }^{18}$ LEIST, a.a.O., 453 f.; STURMA, a.a.O., 226.

19 WEIGEL, S. Genea-Logik. Generation, Tradition und Evolution zwischen Kultur- und Naturwissenschaft, Paderborn 2006, 9, 109; Parense u. a. , Das Konzept der Generation, a.a.O., 11.

20 JUREIT, U. Generationenforschung, Göttingen 2006. 
responsables, actúan inexorablemente en la trama de nexos sociales, posibilitando así la colectivización de su responsabilidad.

Tercero: ambos aspectos, previamente elaborados, apuntan hacia la dimensión sincrónica y también diacrónica del concepto de generación. ${ }^{21} \mathrm{El}$ concepto se convirtió prominente en la sociología, describiendo a grupos de edad específica, que comparten posiciones y estilos de vida comunes. ${ }^{22}$ Generación significa en este contexto una comunidad de acontecimientos y experiencias en el modo de simultaneidad social. Como contraposición a este predominio sociológico se ha enfatizado el aspecto histórico que subyace en el fondo. ${ }^{23}$ Pues generación también significa una sucesión temporal de colectivos sociales que puede ser comprendida como historia cultural. Desde el siglo XVIII se va historizando la idea de una consecución de generaciones dirigiéndola simultáneamente hacia el futuro. El modelo digno en este contexto es el de la herencia cultural, ${ }^{24}$ apropiado para la ética del futuro.

Cuarto: coherente a lo ya mencionado, podemos constatar que el concepto de generación logra mediar entre la corta y larga duración de la historia. La categoría garantiza no solamente actores sociologicamente delimitados, sino también secuencias históricas "medias" en la consecución del tiempo. Esta perspectiva coincide con el propósito de actuar en el presente, considerando plazos planificables y predicables, sin olvidar que cada generación se enfrenta ante su propia definición de metas y propósitos. Cuando se exige, sobre todo, crear condiciones para posibilidades alternas y libres de disposición, se ha de considerar esta oportunidad como una determinación que también han de poder realizar libremente las generaciones venideras. El derecho a la autodeterminación democrática de cada generación presupone el pensamiento en categorías de una cultura determinada de manera biológica y cultural. De esta manera es posible intermediar con el concepeto de generación entre responsabilidad lejana y responsabilidad cercana.

\footnotetext{
21 WEIGEL, S. Genea-Logik, a.a.O., 108; Parense u.a., Das Konzept der Generation, a.a.O., 10 f. 22 MANNHEIM, K. Das Problem der Generation, in: ders.: Wissenssoziologie. Auswahl aus dem Werk, eingeleitet und herausgegeben von Kurz H. Wolf, Berlin, Neuwied, Luchterhand 1964, 509-565. ${ }^{23}$ DILTHEY, W. Über das Studium der Wissenschaft vom Menschen, der Gesellschaft und dem Staat, In: Gesammelte Schriften, Bd. 5, Stuttgart 1964, 35 f. ${ }^{24}$ Cf. WEIGEL, a.a.O., 62.
} 
Las generaciones menudeadas pueden ser comprendidas también como una "cadena de seres vivos", se historizó y proyectó hacia el futuro durante el siglo XVIII. El sentido de ello consiste en crear el sentimiento de solidaridad entre las generaciones, con la exhortación a colaborar para el bienestar de las personas por venir. En el historismo se transfirió la cadena de generaciones hacia el pasado, para así ganar una orientación en la conciencia histórica respecto al tiempo presente. Una identidad histórica de este tipo también se deja transferir a la cadena de generaciones futuras. De la misma manera que se nos apela a desarrollar una postura históricamente fundada hacia Europa, parece ser razonable motivar una conciencia de identidad similar con respecto a temas del futuro. Ante la anonimato de las personas por venir, es necesaria una conciencia de la propia posición en la cadena de las generaciones y un sentimiento colectivo que logre extenderse más allá de la propia generación.

Ciertamente, no es suficiente simplemente creer en la autodinámica de esta "cadena", confiando en que el cuidado inmediato de los padres se trasfiera sin complicaciones hacia los hijos, los cuales han de cuidar de sus propios hijos, de la misma manera que sus padres lo hicieron con ellos. ${ }^{25} \mathrm{El}$ calculo detrás de este planteamiento consiste en suponer que si la generación actual cuida bien de sus niños, los nietos gozaran de mejores condiciones. En vez de simplemente exigir una responsabilidad moral hacia las generaciones venirderas. Sin embargo, con este planteamiento se reduce nuevamente a la ética del futuro a el modelo primario del cuidado familia, como si el problema de una extensión del modelo no hubiese existido en primer lugar. Si el modelo familiar fuese suficiente, estaría de mas buscar una ética para la responsabilidad duradera. De la misma manera que es importante poder aplicar la ética del futuro en una dimensión cotidiana, es importante encontrar un modelo que logre fundar la responsabilidad duradera de alcance medio. Esta exigencia la logra cumplir el modelo de herencia en la sucesión de generaciones.

\section{La herencia}

$\mathrm{Si}$ preguntamos cual es el modo de encadenamiento de las generaciones, podemos constatar, superando la línea biológica en la sucesión

${ }^{25}$ En referencia a PASSMORE lo elabora de tal manera BIRNBACHER, Langzeitverantwortung, a.a.O., $32 \mathrm{f}$. 
de generaciones, que la herencia se puede comprender como una transmisión cultural. El concepto de una herencia cultural es apropiado para delimitar este fenómeno. El concepto de herencia es propicio para rendir la buscada mediación entre las generaciones, puesto que logra superar los angostos limites del cuidado familiar, siendo al mismo tiempo esencialmente más concreto que el entramado de nociones de las teorías universalistas. Además, logra mediar entre naturaleza y cultura, sobre todo porque incorpora la herencia biológica y abarca al mismo tiempo, la herencia cultural. El concepto de herencia se relaciona no sólo con la herencia de bienes materiales, reglamentada de manera jurídica, sino también con los legados culturales. En el contexto de la ética del futuro se deja aplicar este concepto, de modo que da cuenta de la circunstancia, incluso, de que heredamos las condiciones naturales de vida modificadas de manera cultural: También "heredamos" condiciones culturales incluyendo sus recursos naturales.

Herencia significa prácticamente el acontecimiento de entrega por el testador, en el sentido de una concesión, de una continuación, acrecentamiento y cambio por efecto de la herencia. Los conocimientos del ser humano son para Turgot como un tesoro que "se transmite de una generación a la otra como una herencia que se acrecienta con los descubrimientos de cada siglo". ${ }^{26}$ La realidad que surge entre testador y heredero posibilita un uso flexible de la herencia. La herencia no determina la manera de su uso futuro. Su uso queda prendido en la libertad del heredero. La herencia constituye en este sentido la oportunidad en la que se abre una posibilidad de acción. Esto es congruente con el carácter abierto del proceso histórico, dado que respeta el derecho de autodeterminación de cada generación.

La idea elemental en mi propuesta de introducir el concepto de herencia para la fundación de una responsabilidad duradera, consiste en ocupar el tema de manera positiva, posibilitando así un mayor grado de motivación. Si se le instruye a personas constantemente para evitar catástrofes inminentes y salvar a la humanidad, se cumple una exigencia que, a pesar de ser buena, no logra ser realmente motivadora en su calidad de imperativos. Lo que la retórica prescribe, se práctica entonces de manera negativa, de

26 TURGOT, Über die Fortschritte des menschlichen Geistes, hg. Von J. ROHBECK und L. STEINBRÜGGE, Frankfurt a.M. 1990, 140. 
manera que menoscaba la responsabilidad requerida. Si se introdujese el concepto de herencia, se lograría fortalecer el substrato motivación, es decir, la voluntad motivada que conduce a querer dar en herencia los propios logros culturales a las generaciones siguientes. Quien escucha la música de Mozart siente que hay razones para que esta se siga escuchando en el futuro; y quien sabe apreciar el arte de Goya, considera valioso el acto de conservar su obras para que generaciones venideras puedan apreciar el arte a estos niveles. ${ }^{27}$ Mas sin embargo, el modelo de herencia sobrepasa un tal deseo, porque permite también la estructuración de la transmisión cultural. Aca es importante no sola la petición del testador, sino tambien el comportamiento anticipado del que recibe la herencia. Esto es implícito a la práctica del heredar y apunta más allá de las tres generaciones coetáneas.

Es posible reconocer aquí el motivo antropológico de querer extralimitar la muerte individual y encontrar una pervivencia virtual en la herencia cultural. Las personas se oponen a ser olvidadas e intentan con ayuda de la herencia, en cuanto capital simbólico, asegurar su conmemoración en las generaciones futuras. De este modo parece ser posible superar la asimetría en la responsabilidad duradera, puesto que la idea de una contraprestación futura muestra ser prometedora. Surge así la imagen de una relación reciproca entre obligación práctica cercana y una esperada obligación conmemorativa.

La expectativa de la buena conmemoración es un recuerdo anticipado que muestra la estructura temporal del futuro perfecto. En el momento en que las futuras generaciones lleguen a experimentar ciertas consecuencias de nuestras acciones actuales, no solamente seremos responsables por estas acciones, sino que también se nos hará responsables por ellas. De ello puede surgir el deseo de no solamente querer ser responsabilizados por nuestro comportamiento errado, sino también el interés por perdurar en el recuerdo futuro por un acción de cuidado bien realizada. Si formulamos este nexo en categorías de un discurso de memoria transferido hacia el tiempo futuro, podemos hablar de una posible culpa presente con respecto a las victimas del futuro, de mismo modo que las personas por venir nos deberán gratitud, en tanto que no nos hayamos hecho causantes de sus condiciones de vida. El modelo de la herencia concreta, que se transmite de una herencia a la

${ }^{27}$ BIRNBACHER, Langzeitverantwortung, a.a.O., $33 \mathrm{f}$. 
siguiente, podría darle mayor realismo a la figura abstracta de una responsabilidad retrospectiva.

Con la figura de un "recuerdo del futuro" hago referencia nuevamente a la previa fundación de la responsabilidad duradera, en su dimensión temporal e histórica. Espero poder haber demostrado que la filosofía de la historia esta en la disposición de poder enriquecer teóricamente a la ética del futuro. Los teoremas más importantes consistían en la relación de la obligación lejana con el presente, con una delimitación del alcance moral de las obligaciones, así como en la comprensión de la historicidad del presente y del futuro. Ello tenía como consecuencia práctica la comprensión de la relación entre generaciones presentes y futura como una relación simétrica, en el sentido que en la relación práctico con la cultura heredada se han de considerar, de la misma manera que en la generación anterior, con opciones alternativas y libertad en la decisión. El modelo de herencia en la sucesión de generaciones tiene la ventaja de que no solamente logra sobrepasar el modelo de cuidado familiar, sin buscar refugio en destinatarios abstractos, sino que también hace posible imaginar el acto de heredar bienes culturales como un reciproco y contingente nexo de acción. De la misma manera en que la reflexión filosófica obtiene un carácter historico, se logra complementar a la filosofía de la historia por medio de una ética. La meta de la filosofía practica de la historia que propongo, consiste en desprenderse del ámbito de una metodología teórica de la historia para así poder ser parte de la filosofía práctica. 